\title{
NONUNIFORM IRRADIATION OF LASER TARGETS
}

\author{
J.R. SANMARTÍN, J. SANZ and J.A. NICOLÁS
}

Smoothing of plasma ablated from a laser target under weakly nonuniform irradiation is discussed. Conduction is assumed restricted to a quasisteady layer enclosing the critical surface (large pellet or focal spot, and long, low-intensity, short-wavelength pulse). Light refraction can make the ablated plasma unstable.

The direct-drive approach to laser fusion, as opposed to one based on an X-ray hohlraum, has been thought too sensitive to nonuniformities in the driving and to instabilities at the target (ablation) surface. However, new results on Rayleigh-Taylor growth rates and improved optics have made the direct drive more attractive [1]. Hence, the smoothing of nonuniformities in the corona blowing off the target, and any coronal instability, should be thoroughly studied. Smoothing by heat conduction has been discussed in the past [2]; Manheimer et al. included plasma motion in the analysis [3] and Bell and Epperlein discussed magnetic effects and how to deal with the underdense plasma [4].

Here we obtain new results on smoothing and add light refraction to the analysis. We show that refraction is tied up with the smoothing process and may lead to a thermal-focusing instability. As in refs. [3,4] we assume almost uniform irradiation, absorption at critical density $n_{c}$, and conduction restricted to a quasisteady layer lying next to the target and enclosing the critical surface. This requires that its distance $x_{\mathrm{c}}$ to the target be small compared with overall length of plasma $L$ and transverse dimension $R$; transport is then classical [5]. We assume a large ion charge number $Z_{\mathrm{i}}$.

To analyse the layer we use Maxwell's equations, and the equations of continuity and total momentum, and electron entropy and momentum

$\nabla \wedge E=0, \quad \nabla \cdot B=0, \quad \nabla \wedge B=-4 \pi e n u / c$

$$
\begin{aligned}
& \nabla \cdot n \boldsymbol{v}=0, \quad \bar{m} n \boldsymbol{v} \cdot \nabla \boldsymbol{v}=-\boldsymbol{\nabla}(n T), \\
& \frac{3}{2} n(\boldsymbol{v}+\boldsymbol{u}) \cdot \nabla T-T(\boldsymbol{v}+\boldsymbol{u}) \cdot \nabla n \\
& =-\nabla \cdot \boldsymbol{q}-\boldsymbol{u} \cdot \boldsymbol{R}+\boldsymbol{I}^{\prime}, \\
& 0=-\nabla(n T)-e n(E+\boldsymbol{v} \wedge \boldsymbol{B} / c)+\boldsymbol{R} ;
\end{aligned}
$$

$n$ and $T$ are electron density and temperature, $v$ and $\boldsymbol{v}+\boldsymbol{u}$ are ion and electron velocities, $Z_{i} \bar{m}$ the ion mass, $I^{\prime}$ a $\delta$-function term representing laser heating, and $-\boldsymbol{u} \cdot \boldsymbol{R}$ Joule heating. The heat flux is $\boldsymbol{q}=\boldsymbol{q}_{\mathrm{F}}+\boldsymbol{q}_{\mathrm{P}}+\boldsymbol{q}_{\mathrm{RL}}$, and the friction is $\boldsymbol{R}=\boldsymbol{R}_{\mathrm{O}}+\boldsymbol{R}_{\mathrm{S}}+\boldsymbol{R}_{\mathrm{N}} ; \boldsymbol{q}_{\mathrm{F}}$ and $\boldsymbol{R}_{\mathrm{O}}$ are Fourier and Ohm laws, while $\boldsymbol{q}_{\mathrm{P}}, \boldsymbol{q}_{\mathrm{RL}}, \boldsymbol{R}_{\mathrm{S}}$, and $\boldsymbol{R}_{\mathrm{N}}$ represent the Peltier, Righi-Leduc, Seebeck, and Nernst effects [5]. A term -enu $\wedge \boldsymbol{B} / c$, missing from both momentum equations, would be negligible for $\boldsymbol{u}$ and $\boldsymbol{B}$ small as found below; for a similar reason the Ettinghausen and Hall effects have not been considered.

The light is supposed to impinge from the right on a massive target, which thus has negligible acceleration and lies at $x \leqslant 0$. For a uniform absorbed intensity, $I_{0}$, the structure of the layer was determined in the past. It depends only on $x ; \boldsymbol{u}$ and $\boldsymbol{B}$ vanish; and eqs. (2), (3) become

$$
\mathrm{d}\left(n_{0} v_{0}\right) / \mathrm{d} x=0, \quad \mathrm{~d}\left(\bar{m} n_{0} v_{0}^{2}+n_{0} T_{0}\right) / \mathrm{d} x=0,
$$




$$
\begin{aligned}
& \frac{\mathrm{d}}{\mathrm{d} x}\left(n_{0} v_{0}\left(\frac{1}{2} \bar{m} v_{0}^{2}+\frac{5}{2} T_{0}\right)-\bar{K} T_{0}^{5 / 2} \frac{\mathrm{d} T_{0}}{\mathrm{~d} x}\right) \\
& \quad=I^{\prime} \equiv I_{\mathrm{c} 0} \delta\left(x-x_{\mathrm{c} 0}\right) .
\end{aligned}
$$

The equations fail at large $x / x_{\mathrm{c} 0}$, where three-dimensional and (or) unsteady effects are important, conduction negligible, and the flow isentropic. At large $x / x_{\mathrm{c} 0}$ the layer's (inner) solution must match the isentropic (outer) solution at small $x / L\left(x_{\mathrm{co}} / L\right.$ is the small parameter of a singular asymptotic expansion). In general this requires that the isentropic Mach number approach unity as $x / x_{\mathrm{c} 0} \rightarrow \infty$. The surface energy absorption requires an isothermal Mach number unity at $x_{\mathrm{c} 0}$ (where $n_{0}$ and $v_{0}$ have unbounded gradients). The entire structure, eigenvalue $x_{\mathrm{c} 0}$, ablation pressure $P_{\mathrm{a} 0}$, mass ablation rate $\dot{m}_{\mathrm{a} 0}$, etc. follow. For instance, we have $P_{\mathrm{a} 0}=\left(8 / 5^{4 / 3}\right)\left(\bar{m} n_{\mathrm{c}}\right)^{1 / 3} I_{0}^{2 / 3}[6]$.

Consider now a weakly nonuniform absorbed intensity

$I_{\mathrm{c}}=I_{0}+I_{\mathrm{cl}} \exp (\mathrm{i} k y), \quad I_{\mathrm{c} 1} / I_{0} \ll 1$.

The laser term $I^{\prime}$ is then $I_{\mathrm{c}} \delta\left(x-x_{\mathrm{c}}\right)$ where $x_{\mathrm{c}}=x_{\mathrm{c} 0}+$ $x_{\mathrm{c} 1} \exp (\mathrm{i} k y)+\ldots$. A naive expansion of the solution would produce a derivative of the $\delta$-function (and higher singularities) in eq. (3) for first (and higher) order. To avoid this difficulty we define new variables

$s \equiv x\left(1-\mathrm{e}^{\mathrm{i} k y} x_{\mathrm{cl}} / x_{\mathrm{c} 0}+\ldots\right), \quad y \equiv y$,

that is, we strain the $x$-coordinate: $x=s\left(1+\mathrm{e}^{\mathrm{i} k y} x_{\mathrm{c} 1} / x_{\mathrm{c} 0}+\ldots\right)$. Now the critical surface $x=x_{\mathrm{c}}$ lies at $s=x_{\mathrm{c} 0}^{\prime}$ to all orders,

$I^{\prime}=\left(I_{\mathrm{c} 0}+I_{\mathrm{c} 1} \mathrm{e}^{\mathrm{i} k y^{\prime}}\right)\left(1-\mathrm{e}^{\mathrm{i} k{ }^{\prime}} x_{\mathrm{c} 1} / x_{\mathrm{c} O}+\ldots\right) \delta\left(s-x_{\mathrm{c} 0}\right)$.

All variables are now expanded in the form $v_{x}=v_{x 0}(s)+v_{x-1}(s) \exp (\mathrm{i} k y)+\ldots, v_{y}=v_{y !}(s) \exp (\mathrm{i} k y)$ $+\ldots$, and so on. The zeroth order solution $n_{0}(s)$, $v_{x 0}(s), T_{0}(s)$ is given by the same functions of $x\left(n_{0}\right.$, $v_{0}, T_{0}$ ) discussęd above. To next order eqs. (2), (3) yield a fifth order linear system of differential equations with variables coefficients for $n_{1}, v_{x 1}, v_{y 1}$, and $T_{0}$, coupled to a second order equation for current $n_{0} u_{x: 1}$, obtained from (1) and (4); the entire system contains one eigenvalue, $x_{\mathrm{cl}}$. Eight conditions are needed for the solution:

(1)-(4) At the target $(s=0)$, bounded pressure

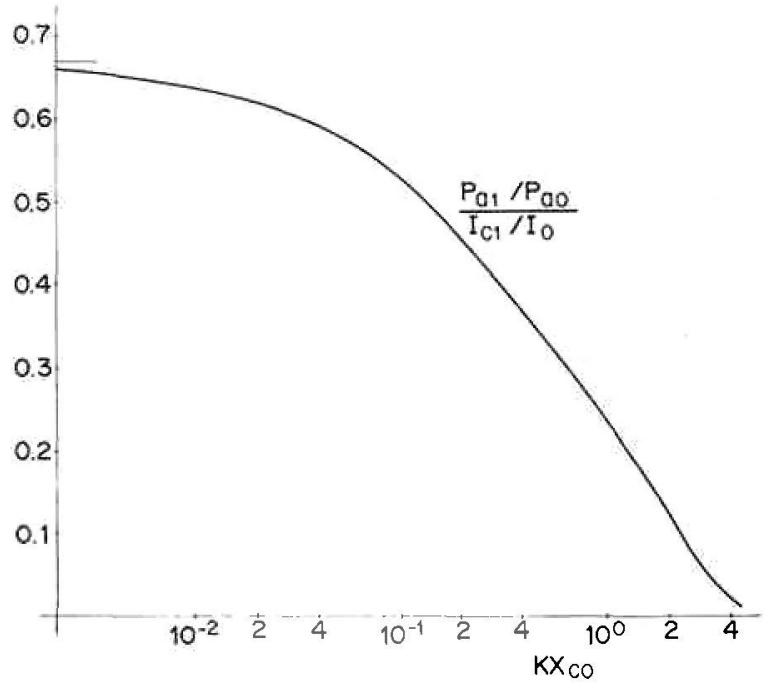

Fig. 1. Fractional perturbed ablation pressure normalized with fractional perturbed absorbed intensity for laser wavelength large compared with electron mean free path; $k=$ perturbation wavenumber, $x_{\mathrm{c} 0}=$ distance to critical surface.

and mass flow rate and continuous $v_{y l}$, leading to $T_{1}=v_{x 1}=v_{y 1}=0$ : also, zero charging current, $n_{0} u_{x 1}=0$.

(5), (6) At the critical surface $\left(s=x_{\mathrm{co}}\right)$, vanishing $n_{1}\left(n-n_{0}\right.$ vanishes to all orders) and bounded fluid variables (one mode of the fifth order system is singular at $s=x_{\mathrm{c} 0}$ because $v_{0}$ is sonic there).

(7), (8) As $s / x_{\mathrm{c} 0} \rightarrow \infty$, bounded current and fluid variables (both the fifth and second order systems have one unbounded mode each).

The solution depends on $k x_{\mathrm{c} 0}$ and on the ratio $A$ of electron mean free path to laser wavelength (characterizing magnetic effects). Figs. 1 and 2 show perturbed ablation pressure $P_{\mathrm{al}}$ and critical surface location $x_{\mathrm{cl}}$, in normalized form for $A$ small (results that neglect magnetic effects altogether are practically the same). Note first that as $k x_{\mathrm{c} 0} \rightarrow 0$, the respective ordinates go to $2 / 3$ and $4 / 3$, as they should from the laws $P_{\mathrm{a} 0} \propto I_{0}^{2 / 3}$ (previously mentioned) and $x_{\mathrm{c} 0} \propto I_{0}^{4 / 3}$; another behavior was found in ref. [4]. Second, $x_{\mathrm{cl}} / I_{\mathrm{cl}}$ is negative for $k x_{\mathrm{c} 0} \gtrsim 1$ : At such wavenumbers, a large lateral energy flux will require a substantial overdense longitudinal gradient; if the increase of critical temperature with laser intensity is insufficient, critical and ablation surfaces get closer so as to sustain that gradient. 


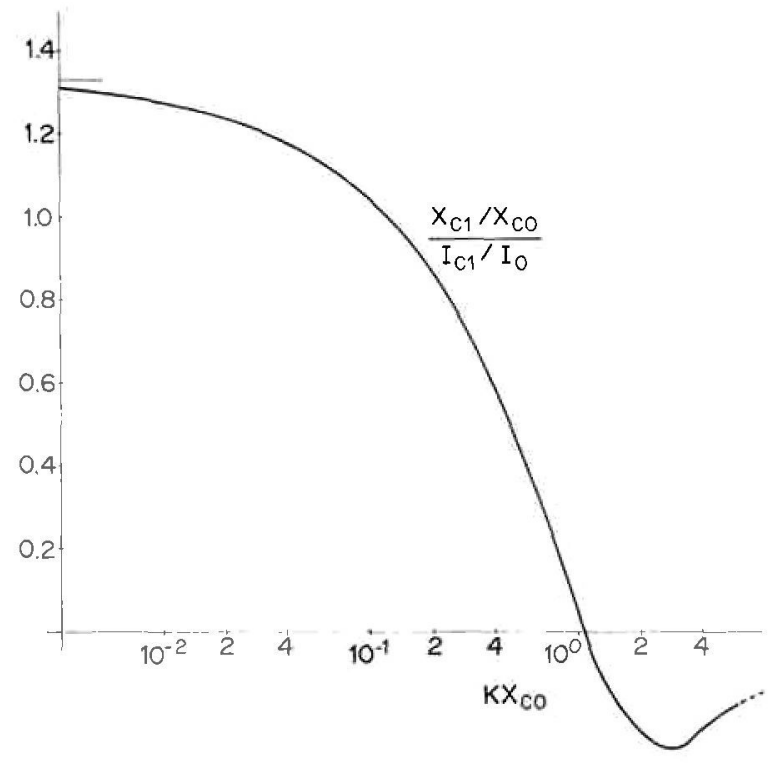

Fig. 2. Normalized perturbed distance to critical surface.

The perturbed density varies transversely, leading to refraction in the range $1<s / x_{\mathrm{c} 0}<\infty$. If $\epsilon$ is the dielectric function $\left(1-n / n_{\mathrm{c}}\right), \tau$ the unit vector along a ray, and $I(s, y)$ the light intensity, the propagation equations are [7]

$\tau \cdot \nabla \tau=\frac{1}{2 \epsilon}[\nabla \epsilon-\tau(\tau \cdot \nabla \epsilon)], \quad \nabla \cdot(\tau I)=0$.

Setting $I=I_{0}+I_{1}(s) \mathrm{e}^{\mathrm{i} k y^{\prime}}+\ldots, \quad \tau_{y}=\tau_{y 1}(s) \mathrm{e}^{\mathrm{i} k y}+\ldots$ and using boundary conditions $I_{1}=I_{c 1}$ at $s=x_{\mathrm{c} 0}, \tau_{p 1} \rightarrow 0$ as $s / x_{\mathrm{c} 0} \rightarrow \infty$ (parallel ray incindence) we get $I_{\mathrm{c} 1}-I_{\infty 1}=\alpha I_{\mathrm{c} 1}$, with $\alpha$ obtained from the $n_{1}(s)$ profile previously determined. Fig. 3 shows $I_{\mathrm{c} 1} / I_{\infty}$, which comes off negative $(\alpha>1)$ at low $k x_{\mathrm{c} 0}$. A positive $\alpha$ implies refraction into $y$-ranges with absorbed intensity above the mean: at low $k x_{\mathrm{co}}$ transverse conduction is inefficient, and though such a $y$-range has a near underdense plasma hotter and denser than neighbor ranges, further away the transverse density gradient is inverted because of lateral pressure forces. An instability analysis (where $I_{\infty 1}=0$ ), now in progress, should show the corona unstable at low $k x_{\mathrm{c} 0}$ since refraction would feedback any perturbation in the absorbed intensity. This is a thermal focusing instability. Estabrook et al. [8] carried out simulations at low $n / n_{\mathrm{c}}$ and no magnetic field.

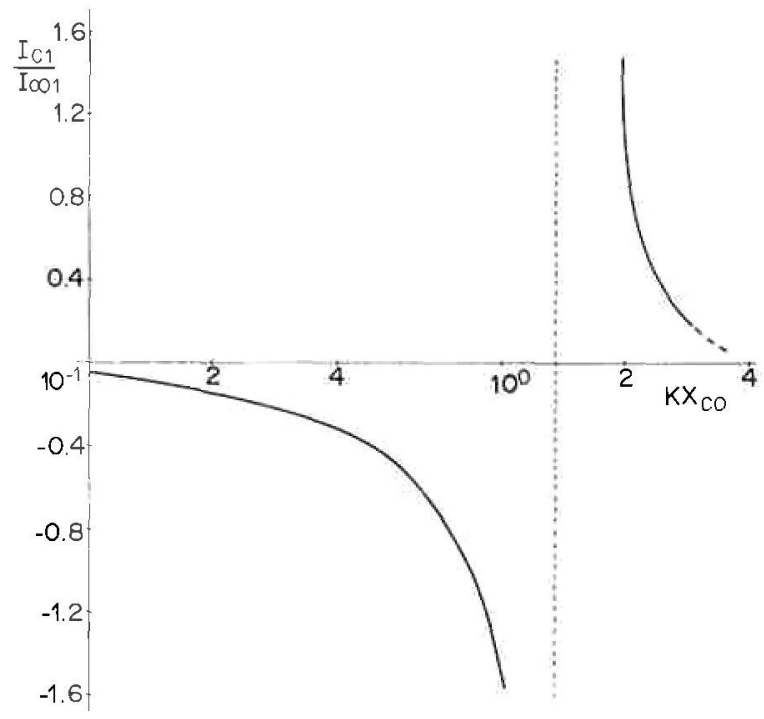

Fig. 3. Critical-to-incident perturbed intensity ratio.

Present results are clearly just indicative. Fig. 3 is only valid for $k$ not too low: $k x_{\mathrm{c} 0} \gg x_{\mathrm{c} 0} / R$, and $k x_{\mathrm{c} 0} \gg\left(x_{\mathrm{c} 0} / L\right)^{1 / 2}$ (we found $n_{1} \sim \exp \left(-k^{2} x_{\mathrm{c} 0} s\right)$ for small $k$ and large $s)$. The neglect of inverse bremsstrahlung at the long, low-intensity, short-wavelength pulses required for conduction to be restricted to a layer is unrealistic. Three-dimensional and unsteady effects, higher intensities, and then fluxlimited conduction, need consideration. Such work is now in progress.

This work was supported by the Comision Asesora de Investigación Científica-Técnica (Project 1983/82 ) and the Instituto de Fusión Nuclear of Spain.

\section{References}

[1] J.H. Gardner and S.E. Bodner, Phys. Fluids 29 (1986) 2672.

[2] J. Nuckolls, L. Wood, A. Thiessen and G. Zimmerman, Nature 239 (1972) 139;

J.H. Gardner and S.E. Bodner, Phys. Rev. Lett. 47 (1981) 1137.

[3] W.M. Manheimer, D.G. Colombant and J.H. Gardner, Phys. Fluids 25 (1982) 1644.

[4] A.R. Bell and E.M. Epperiein, Plasma Phys. Controll. Fusion 28 (1986) 897. 
[5] S.I. Braginskii, in: Review of plasma physics, Vol. 1 (Consultants Bureau, New York, 1965).

[6] J.R. Sanmartín and A. Barrero, Phys. Fluids 21 (1978) 1957; A. Barrero and J.R. Sanmartín, Plasma Phys. 22 (1980) 617; J. Sanz, A. Liñán, M. Rodríguez and J.R. Sanmartín, Phys. Fluids 24 (1981) 2098;
J.R. Sanmartín, J.L. Montañés and A. Barrero, Phys. Fluids 26 (1983) 2754.

[7] L. Landau, Electrodynamics of continuous media (Pergamon, New York, 1980).

[8] K. Estabrook, W.L. Kruer and D.S. Bailey, Phys. Fluids 28 (1985) 19. 